\title{
Fast quasi-conformal regional flattening of the left atrium
}

\author{
Marta Nuñez-Garcia ${ }^{\mathrm{a}, *}$, Gabriel Bernardino ${ }^{\mathrm{a}, \mathrm{b}}$, Francisco Alarcón ${ }^{\mathrm{c}, \mathrm{d}}$, Gala Caixal $^{\mathrm{c}, \mathrm{d}}$, Lluís Mont $^{\mathrm{c}, \mathrm{d}}$, Oscar Camara ${ }^{\mathrm{a}}$, \\ Constantine Butakoff ${ }^{\mathrm{a}}$ \\ ${ }^{a}$ Physense, Department of Information and Communication Technologies, Universitat Pompeu Fabra, Barcelona, Spain \\ ${ }^{b}$ Philips Research, Medisys, France \\ ${ }^{c}$ Department of Cardiology, Unitat de Fibrillació Auricular(UFA), Hospital Clinic, Universitat de Barcelona, Spain \\ 'Institut d'Investigacions Biomèdiques August Pi i Sunyer (IDIBAPS), Barcelona, Spain
}

\begin{abstract}
Two-dimensional representation of 3D anatomical structures is a simple and intuitive way for analysing patient information across populations and image modalities. It also allows convenient visualizations that can be included in clinical reports for a fast overview of the whole structure. While cardiac ventricles, especially the left ventricle, have an established standard representation (e.g. bull's eye plot), the 2D depiction of the left atrium (LA) is challenging due to its sub-structural complexity including the pulmonary veins (PV) and the left atrial appendage (LAA). Quasiconformal flattening techniques, successfully applied to cardiac ventricles, require additional constraints in the case of the LA to place the PV and LAA in the same geometrical 2D location for different cases. Some registrationbased methods have been proposed but 3D (or 2D) surface registration is time-consuming and prone to errors if the geometries are very different. We propose a novel atrial flattening methodology where a quasi-conformal 2D map of the LA is obtained quickly and without errors related to registration. In our approach, the LA is divided into 5 regions which are then mapped to their analogue two-dimensional regions. A dataset of 67 human left atria from magnetic resonance images (MRI) was studied to derive a population-based 2D LA template representing the averaged relative locations of the PVs and LAA. The clinical application of the proposed methodology is illustrated on different use cases including the integration of MRI and electroanatomical data.
\end{abstract}

Keywords: left atrium, two-dimensional map, conformal flattening, regional flattening

\section{Introduction}

Medical imaging techniques are increasingly used nowadays to extract crucial anatomical and functional information used in diagnosis and treatment planing. The imaged organs are inherently three-dimensional and appropriate visualization tools are needed, including manual rotations and different points of view, to get a complete overview of the whole organ under study.

Flattening methods aim to compute an unfolded representation of a $3 \mathrm{D}$ surface mesh by projecting it to a simpler 2D domain, easier to visualize, manage and interpret. Additionally, if the 2D map is standardised (e.g. same anatomical regions of different subjects spatially coincide) the 2D unfolded domains can be used as a common reference space to analyse multi-modal

${ }^{*}$ Corresponding author at: DTIC, Universitat Pompeu Fabra, Carrer de Tànger, 122-140, 08018, Barcelona, Spain.

Email address: marta. nunez@upf .edu (Marta Nuñez-Garcia ) data from different patients or from the same patient at different time-steps. The reader is referred to Kreiser et al. (2018) for a thorough review of flattening methods applied to human organs, including the brain, different bones, and the vascular system among others.

In the case of the heart, the 17 segment AHA bull's eye plot of the left ventricle (LV) has been widely used by clinicians for long time (Cerqueira et al., 2002, SotoIglesias et al., 2016, Paun et al., 2017). The LV's conical shape and the absence of salient sub-structures in $3 \mathrm{D}$ anatomies derived from medical images (e.g. ignoring the trabeculations) highly facilitates its flattening. In general, only one basal boundary is considered, which is mapped to the outer contour of a $2 \mathrm{D}$ disk, and one reference point (the apex) which is placed in the centre of the circle. On the contrary, unfolding of the LA is challenging since its main cavity is connected to several pulmonary veins, the left atrial appendage and the left ventricle through the mitral valve (MV). Furthermore, 
there exists a notable variability in the anatomical configuration and spatial location of these sub-structures. For instance, the most common LA morphology (up to $70 \%$ ) involves $4 \mathrm{PV}$ (with variable size, shape, position and orientation with respect to the main cavity) with occasional presence of a common left trunk, extra right PV or other oddities (Prasanna et al., 2014). Strong variation can also be seen in the LAA shape and the MV size, shape and position with respect to the PVs (García-Isla et al. (2018). Proposing a common reference space for the LA, including the multiple possible interfaces between the main cavity and its sub-structures, is therefore non trivial.

Ma et al. (2012) were the first researchers proposing a LA flattening technique, based on a B-spline with proportional distance between any pair of points in the 3D LA surface and their mapped pairs on the 2D map. As a result, the different $2 \mathrm{D}$ maps were not standardised since the position of the PV holes were not in the same position for different patients. Karim et al. (2014) proposed to flatten the LA to a square also without constraining the position of the PV and LAA holes. The authors carefully studied the distortion induced by the unfolding process and showed how the LA flattened square could be used to display and qualitatively analyse different types of data from the same patient. However, a direct comparison across different patients was not feasible because of the lack of correspondence between the different maps (e.g. PV holes were not positioned in the same place). More recently, Williams et al. (2017) proposed a standardised unfold map (SUM) suitable to compare different LA using as reference a 3D LA template and its corresponding 2D flattened version, both related with a defined 3D-2D point-to-point mapping. Their flattening strategy was based on registering an arbitrary LA to the 3D template and then transferring the data to the 2D LA template with the known 3D-2D template mapping. The original LA template was divided into 24 regions, allowing regional analysis of the mapped data. The main limitation of this approach is the difficulty of obtaining an accurate registration between different LA surface meshes due to their high shape variability. Together with errors induced by required data projection and interpolation steps, this scheme leads to undesired information loss between the 3D and 2D LA representations if the LA under study is substantially different from the LA template. Additionally, advanced surface mesh registration techniques such as currents-based ones are associated to large computational times.

In this paper, we propose a quasi-conformal (in general, there is no conformal map compatible with a given map along the boundary) flattening of the LA with the following boundary constraints: the MV contour is mapped to the external circumference of a 2D disk, and the PVs and LAA ostia contours are mapped to predefined circumferences within the disk. Quasiconformal flattening of surface meshes with holes often results in undesired mesh self-folding (holes appear covered by adjacent mesh cells) and to overcome this issue, we impose additional regional constraints. Five anatomical regions are defined in the $3 \mathrm{D}$ LA which are afterwards confined to their 2D counterparts. Our method is fast (almost real-time) and without information loss (all points in the 3D mesh are represented in the $2 \mathrm{D}$ domain). In order to define a realistic twodimensional template, the relative position of the PVs and LAA as well as the different PV and LAA ostium sizes need to be considered. We computed the average position of these sub-structures analysing a population of 67 atrial shapes and used them to define an intuitive and realistic unfolded representation of the LA, that at the same time minimizes the distortion inherent to any flattening technique. To illustrate the proposed methodology we used several synthetic datasets, real LA from Late-Gadolinium Enhanced Cardiac Magnetic Resonance (LGE-CMR) data and real electroanatomical maps.

\section{Methods}

A scheme of the complete flattening framework can be seen in Figure 1 and it is described in section 2.1. The methodology employed to define the most appropriate $2 \mathrm{D}$ template is detailed in section 2.2

\subsection{Flattening pipeline}

The method comprises three main steps: (1) LA cavity extraction; (2) boundary and regional constraints definition; and (3) Fast Regional Flattening (FRF).

\subsubsection{LA cavity extraction}

Given any arbitrary 3D surface mesh representing a LA, the first step aims at standardising the LA shape by only keeping its main cavity after semi-automatically cutting the PVs, the LAA and the MV. This cutting process requires to manually place 5 seeds near the ending points of the PVs and the LAA. The reader is referred to Tobon-Gomez et al. (2015) for more details on this method. 


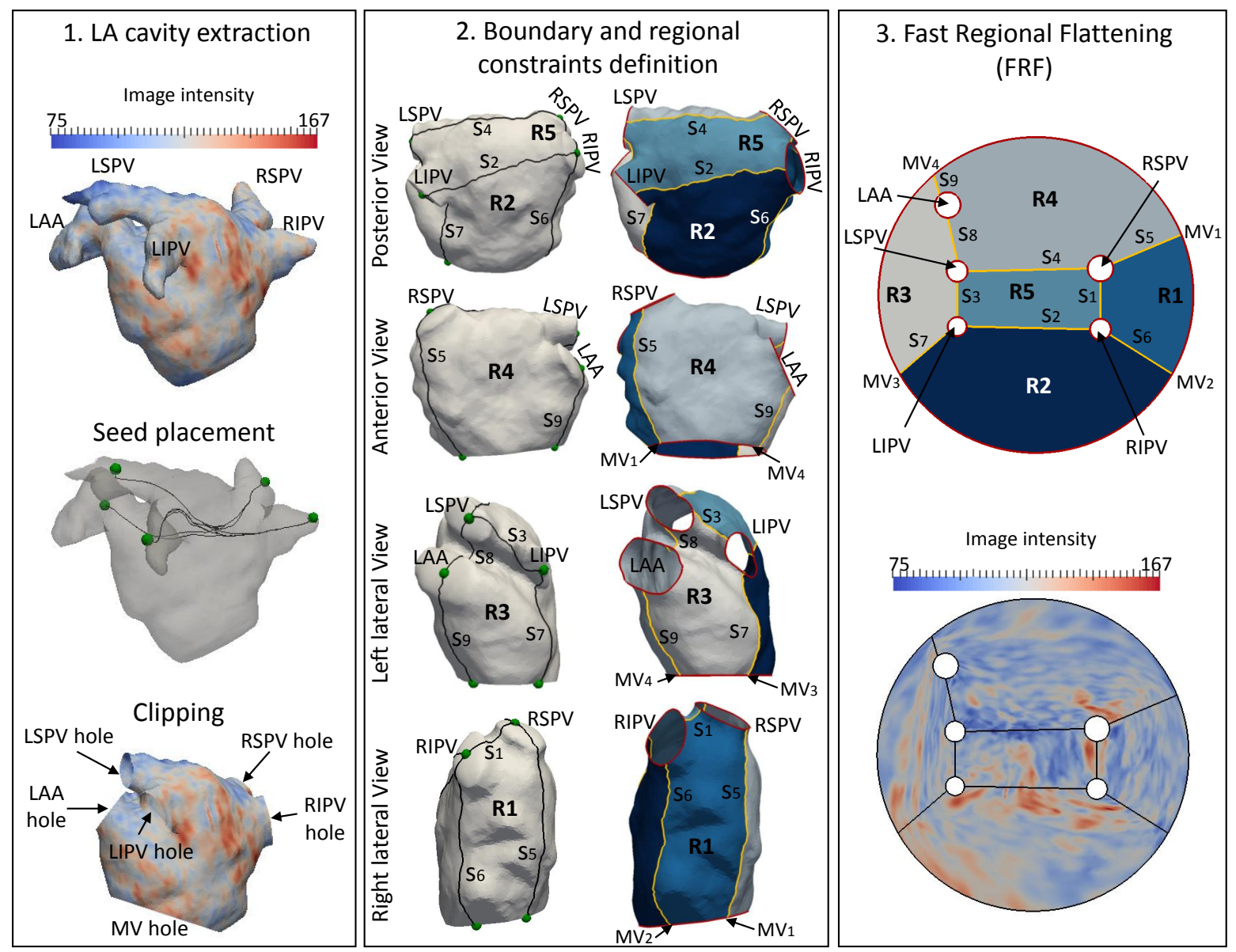

Figure 1: Overview of the proposed method: (1) LA cavity extraction; (2) boundary and regional constraints definition (LA division); (3) Fast Regional Flattening (FRF). Six boundaries (displayed in red) corresponding to the MV, 4 PV, and LAA ostia are used. Nine segment paths ( $\mathrm{s}_{1-9}$, in yellow) are used to define 5 LA regions (R1-R5). Data mapped into the LA surface mesh correspond to intensity values from the associated late-gadolinium magnetic resonance image (blue and red colors indicate low and high image intensity, respectively). LSPV $=$ left superior PV;

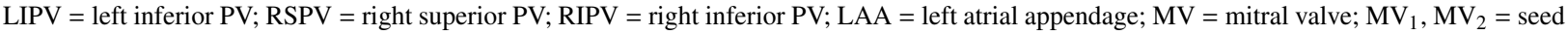
points in the $\mathrm{MV}$ contour delimiting the septal wall, and $\mathrm{MV}_{3}, \mathrm{MV}_{4}=$ seed points in the $\mathrm{MV}$ contour delimiting the left lateral wall.

\subsubsection{Boundary and regional constraints definition}

In the second stage, holes in the main LA cavity corresponding to interfaces with the PV and the LAA are closed and triangulated using the method described in Liepa (2003). Then, 9 seed points are manually placed in specific atrial regions: 5 at the centre of the closed PV and LAA ostia, and other 4 on the MV contour: 2 delimiting the interatrial septal wall and 2 delimiting the left lateral wall. Inter-seed geodesic paths (i.e. shortest curve between two points on a mesh, such that the curve lies on the surface (Mitchell et al., 1987), $\mathrm{s}_{1}-\mathrm{s}_{9}$ in Figure 1) are computed dividing the LA surface into 5 anatomical regions (R1-R5 in Figure 1):

- R1 defines the inter-atrial septal wall, which is delimited by inter-seed geodesic connecting the 2 right PVs $\left(\mathrm{s}_{1}\right)$, geodesics connecting them with the MV septal seeds ( $\mathrm{s}_{5}$ and $\mathrm{s}_{6}$ ), and the corresponding piece of $\mathrm{MV}$ contour between $\mathrm{MV}_{1}$ and $\mathrm{MV}_{2}$.

- R2 corresponds to the part of the posterior wall and LA floor delimited by the 2 inferior PVs $\left(s_{2}\right)$, the geodesics connecting them with the MV contour $\left(s_{6}\right.$ and $\left.s_{7}\right)$, and the $\mathrm{MV}$ contour between $\mathrm{MV}_{2}$ and $\mathrm{MV}_{3}$.

- R3 corresponds to the left lateral wall and it is delimited by the geodesics connecting the MV contour with the LAA $\left(s_{9}\right)$, the LAA with the LSPV $\left(s_{8}\right)$, the two left PVs $\left(s_{3}\right)$, the LIPV with the MV contour $\left(\mathrm{s}_{7}\right)$, and the $\mathrm{MV}$ contour between $\mathrm{MV}_{3}$ and $\mathrm{MV}_{4}$. 


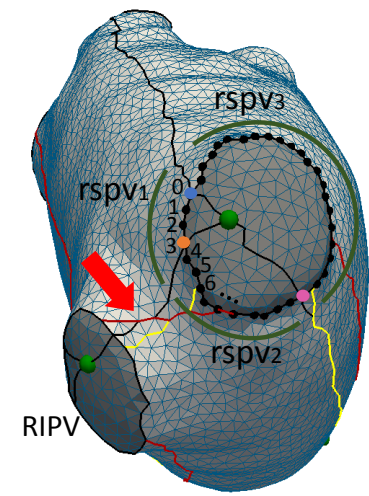

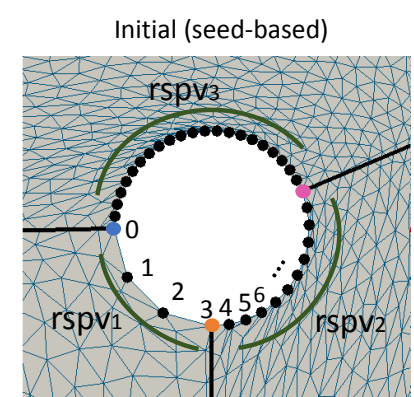

(a)
Sub-contours division

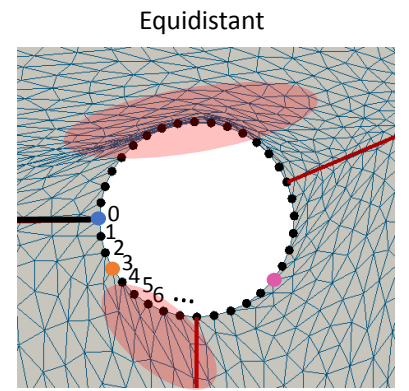

(b)

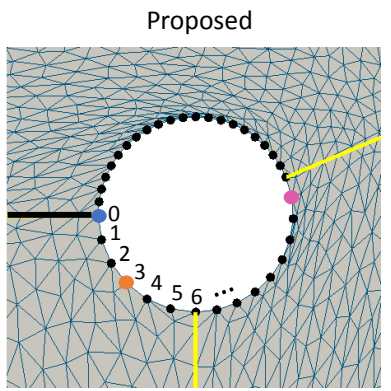

(c)

Figure 2: Constraints recomputation. On the left, 3D LA surface mesh (RSPV detail) with different divisions considered: initial seed-based division (black paths); division achieved imposing equidistant points in the hole (red paths); and our proposed division (yellow paths). Contour points (vertices) are enumerated, and initial sub-contour extremes (intersection points) are shown in blue (fixed reference point), orange and pink. On the right, flattened RSPV ostium contour using the original geodesic paths (a), recomputed paths using equidistant points (b) and recomputed paths using the proposed division (c). The RSPV point seed (green) is slightly displaced to the border of the RSPV ostium to exaggerate this effect in the example. $\mathrm{rspv}_{i}=i$-th sub-contour of RSPV ostium contour; RSPV $=$ right superior PV.

- R4 defines the anterior wall, which is bounded by geodesics connecting the MV contour and the RSPV $\left(s_{5}\right)$, both superior veins $\left(s_{4}\right)$, the LSPV and LAA $\left(s_{8}\right)$, the LAA and MV contour $\left(s_{9}\right)$, and the $\mathrm{MV}$ contour between $\mathrm{MV}_{4}$ and $\mathrm{MV}_{1}$.

- R5 corresponds to the part of the posterior wall framed by the geodesics connecting the 4 PVs $\left(s_{1-4}\right)$.

After that, the final LA cavity that will be flattened is obtained by automatically removing the PV and LAA hole covers. The boundary constrained points are identified as the PV, LAA and MV boundary points (mesh vertices of the red curves in Figure 1, and the regional constrained points are identified as the projection of the inter-seed paths $\left(\mathrm{s}_{1}-\mathrm{s}_{9}\right)$ onto the final LA cavity (points of the yellow curves in Figure 11.

In our framework, the PV and LAA ostium contours are mapped to discretised circumferences and their correct representation depends on the sampling (total number of vertices or points) of the ostia contours: a small number of points induces circumferences with large edges, while a high number of points produces circumferences with very short edges. The LAA and PV ostia contours are actually divided into 2 and 3 sub-contours, respectively, as a consequence of the LA regional division explained above, further influencing how the circumferences are represented in the 2D domain. Initially, these sub-contours are determined by the inter-seeds paths which likely cause non-equidistant point distribution in the contour (Figure 27). An equidistant distribution of points (Figure
$2 \mathrm{p})$ can be achieved by conveniently displacing the subcontour point extremes (or intersection points: blue, orange and pink dots in Figure 2), but this could potentially worsen the anatomical meaning of the LA division (note the red arrow showing how the red line connecting RSPV and RIPV is twisted), and induce mesh distortion (see areas with stretched triangles (highlighted with semi-transparent red ellipses in Figure 2p). Defining as proportional length the number of points of each sub-contour corresponding to an equidistant distribution of points in the complete hole, and real length as the number of points of the sub-contour derived from the initial LA division, a good compromise between suitable distribution of points and meaningful LA division is achieved by imposing a sub-contour length equal to real length $+\lfloor$ (proportional length - real length $) / 2\rfloor$ number of points. Note that the second addend can be either positive or negative. One intersection point is fixed (point with index 0 and blue color in Figure 2) and the remaining two are updated using the previous rule. This modifies the number of points in each sub-contour but the total number of points remains the same. Using the updated sub-contour extremes, the boundary and regional constrained points are recomputed.

\subsubsection{Fast Regional Flattening (FRF) \\ Overview}

We aim to obtain a quasi-conformal (i.e. anglepreserving) and standardised flat representation of the 3D LA cavity surface mesh where the boundaries (MV, PV and LAA holes) are constrained to predefined circumferences within the 2D disk. An example of con- 


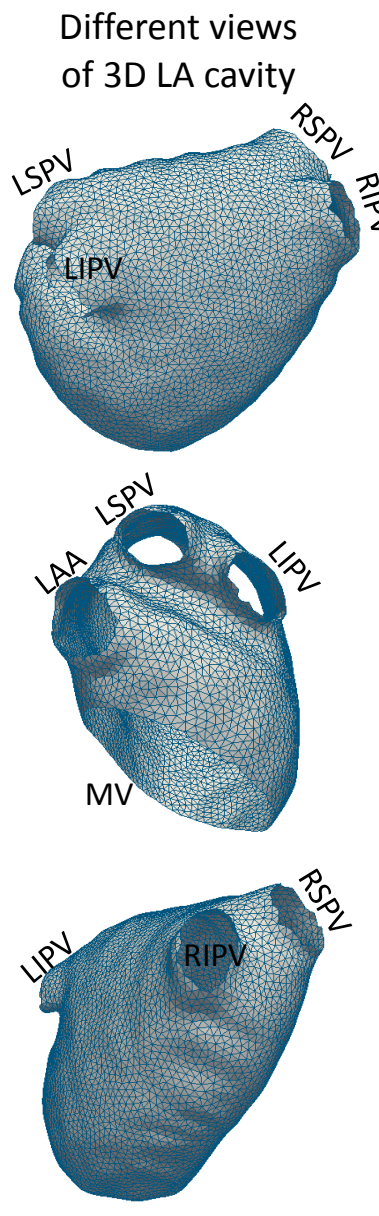

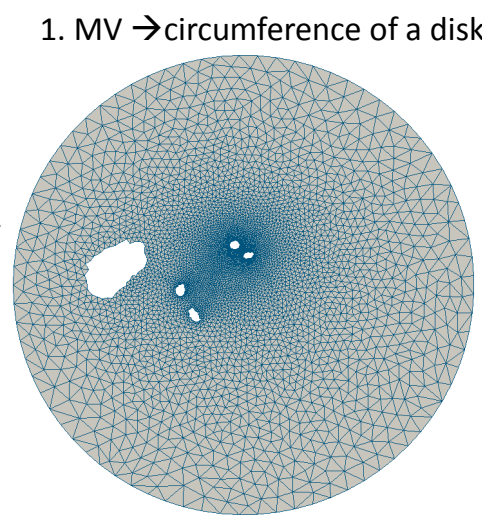

(a)

1. $M V \rightarrow$ circumference of a disk 2. PV and LAA $\rightarrow$ predefined holes 3. Additional regional constraints

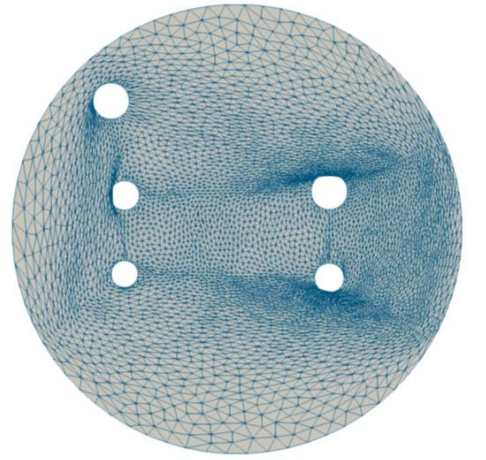

(c)
1. $\mathrm{MV} \rightarrow$ circumference of a disk

2. PV and LAA $\rightarrow$ predefined holes

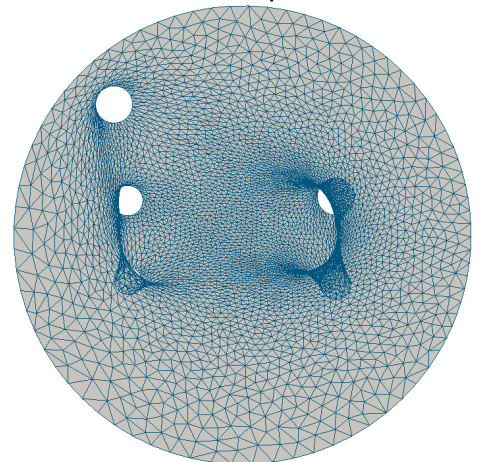

(b)

1. $\mathrm{MV} \rightarrow$ circumference of a disk

2. PV and LAA $\rightarrow$ predefined holes

3. Additional regional constraints

4. Boundary refinement

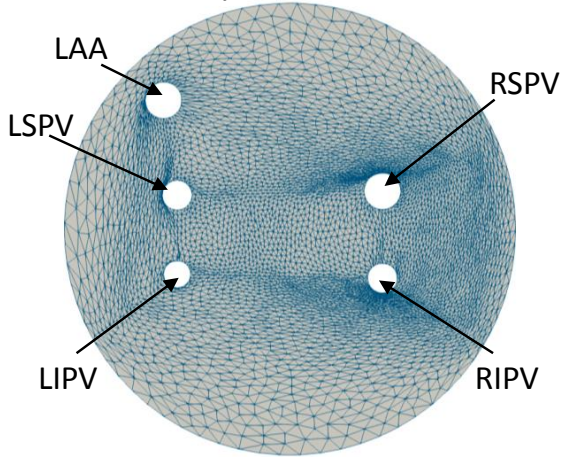

(d)

Figure 3: Example of 3D LA surface mesh (left column, 3 different views) and different LA unfolding strategies: (a) quasi-conformal flattening constraining only the MV to the external boundary of a 2D disk; (b) quasi-conformal flattening additionally fitting the PV and LAA hole contours; (c) quasi-conformal flattening fitting PV, LAA contours and imposing regional constraints; (d) final flattening after boundary refinement of (c) mesh. LSPV = left superior PV; LIPV = left inferior PV; RSPV = right superior PV; RIPV = right inferior PV; LAA = left atrial appendage; MV $=$ mitral valve.

formal LA flattening where only the MV boundary position is imposed (i.e. only MV boundary is mapped to the circumference of the 2D disk) can be seen in Figure 3a. All holes except the MV were closed prior to the flattening and opened afterwards. In order to additionally restrict the PV and LAA ostia contours to predefined positions we add the corresponding boundary constraints to the quasi-conformal scheme. By doing that, the boundary points appear correctly placed in the targeted positions but the holes are often covered by adjacent triangles since the method does not avoid mesh self-folding (Figure $3 \mathrm{~b}$ ). To overcome this issue, we include additional regional constraints in the parameterisation, but minor triangle overlapping can still occur near the holes (Figure 3r). To further refine the boundary we recompute the point coordinates on the $2 \mathrm{D}$ flattened LA with a quasi-conformal parameterisation only constrained with the boundary points and not the regional constraints (Figure 3 $\mathrm{d}$ ).

\section{Mathematical framework}

Let $M$ be the LA cavity surface mesh with $\mathrm{N}$ vertices (points) and $M_{F}$ its corresponding flattened mesh. Let $I_{\partial M}$ be the indices of the boundary points (MV, PV, LAA) of $M$. Let $\Delta_{M}$ be the $N \times N$ (cotangent (Pinkall and Polthier. 1993) Laplacian of $M$ and let $\Delta_{M}^{\prime}$ be $\Delta_{M}$ where the off-diagonal elements of the rows defined by the positions of $I_{\partial M}$ set to 0 and corresponding elements 


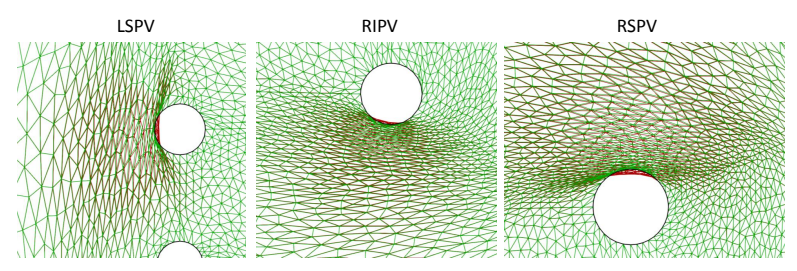

Figure 4: Zoom of PV holes region after the initial flattening (red) and after boundary refinement (green). Target boundaries are shown in black. LSPV = left superior PV; RIPV = right inferior PV; RSPV right superior PV.

on the main diagonal set to 1 . Let $\left(b_{x}, b_{y}\right)$ be the coordinates of the 2D boundary points of $M_{F}$ in the same order as the corresponding indices appear in $I_{\partial M}$ and let $\boldsymbol{b}_{x}^{\prime}$ and $\boldsymbol{b}_{y}^{\prime}$ be $\mathrm{N}$-dimensional vectors with values $\boldsymbol{b}_{x}$ and $\boldsymbol{b}_{y}$, respectively, in the positions of $I_{\partial M}$ and zeros elsewhere.

Let $\mathrm{P}$ be the number of regional constrained points (i.e. number of points in the dividing segments $\left(\mathrm{s}_{1}-\mathrm{s}_{9}\right)$, yellow lines in Figure 1 and $I_{s}$ the corresponding point (vertex) indices. Let $\left(s_{x}, s_{y}\right)$ be the $2 \mathrm{D}$ coordinates of the $\mathrm{P}$ vertices in the same order as they appear in $I_{s}$ and let $\boldsymbol{E}_{s}$ be a $P \times N$ zero matrix with 1 in each row in the positions corresponding to vertices of $I_{s}$ (i-th row has 1 in the position given by the i-th element of $\left.I_{s}\right)$.

In order to find the coordinates $\left(\boldsymbol{x}^{*}, \boldsymbol{y}^{*}\right)$ of the vertices of $M_{F}$, we propose to solve the following two quadratic programming problems:

$$
\begin{array}{lll}
\boldsymbol{x}^{*}=\arg \min _{\boldsymbol{x}}\left(w\left\|\boldsymbol{\Delta}_{M}^{\prime} \boldsymbol{x}-\boldsymbol{b}_{x}^{\prime}\right\|^{2}\right) & \text { s.t. } & \boldsymbol{E}_{s} \boldsymbol{x}=\boldsymbol{s}_{x} \\
\boldsymbol{y}^{*}=\arg \min _{\boldsymbol{y}}\left(w\left\|\boldsymbol{\Delta}_{M}^{\prime} \boldsymbol{y}-\boldsymbol{b}_{y}^{\prime}\right\|^{2}\right) & \text { s.t. } & \boldsymbol{E}_{s} \boldsymbol{y}=\boldsymbol{s}_{y}
\end{array}
$$

with $w$ (set to 1000 in our experiments) penalizing the unfulfilment of the boundary constraints. Using Lagrange multipliers these can be rewritten and solved as a system of linear equations:

$$
\begin{aligned}
& {\left[\begin{array}{cc}
w^{2} \boldsymbol{\Delta}_{M}^{\prime} \boldsymbol{\Delta}_{M}^{\prime} & \boldsymbol{E}_{s}^{T} \\
\boldsymbol{E}_{s} & \mathbf{0}
\end{array}\right]\left[\begin{array}{c}
\boldsymbol{x}^{*} \\
\boldsymbol{\lambda}_{x}
\end{array}\right]=\left[\begin{array}{c}
w^{2} \boldsymbol{\Delta}_{M}^{\prime T} \boldsymbol{b}_{x}^{\prime} \\
\boldsymbol{s}_{x}
\end{array}\right]} \\
& {\left[\begin{array}{cc}
w^{2} \boldsymbol{\Delta}_{M}^{\prime} \boldsymbol{\Delta}_{M}^{\prime} & \boldsymbol{E}_{s}^{T} \\
\boldsymbol{E}_{s} & \mathbf{0}
\end{array}\right]\left[\begin{array}{c}
\boldsymbol{y}^{*} \\
\boldsymbol{\lambda}_{y}
\end{array}\right]=\left[\begin{array}{c}
w^{2} \boldsymbol{\Delta}_{M}^{\prime T} \boldsymbol{b}_{y}^{\prime} \\
\boldsymbol{s}_{y}
\end{array}\right]}
\end{aligned}
$$

To refine the boundary we proceed as follows. Let $\Delta_{F}$ be the $N \times N$ Laplacian of $M_{F}$ and let $\Delta_{F}^{\prime}$ be $\Delta_{F}$ where the off-diagonal elements of the rows defined by the positions of $I_{\partial M}$ set to 0 and corresponding elements on the main diagonal set to 1 . The refined coordinates

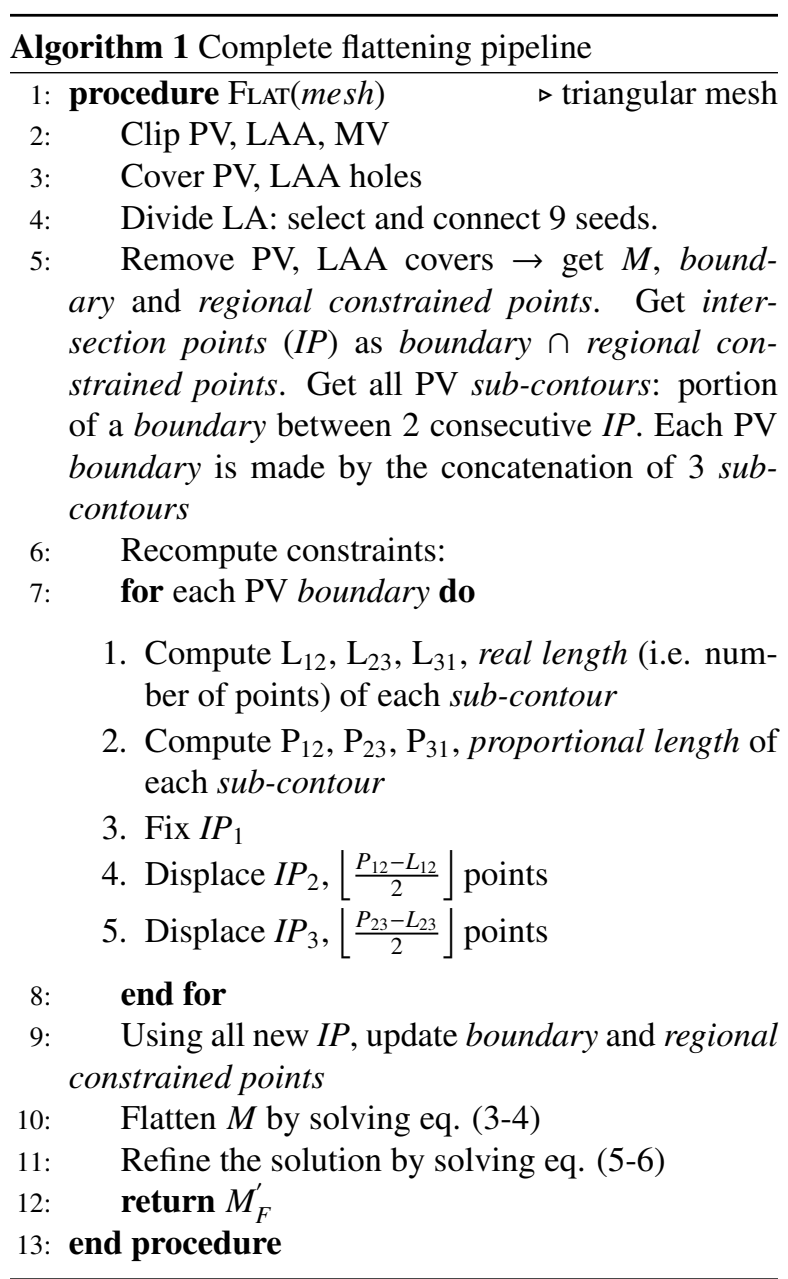

$\left(\boldsymbol{x}^{*^{\prime}}, \boldsymbol{y}^{*^{\prime}}\right)$ of the vertices of $M_{F}$ in the final flat representation can be found by solving the following system of linear equations:

$$
\begin{aligned}
\Delta_{F}^{\prime} x^{*^{\prime}} & =b_{x}^{\prime} \\
\Delta_{F}^{\prime} y^{*^{\prime}} & =b_{y}^{\prime}
\end{aligned}
$$

The need for this boundary refinement is illustrated in Figure 4 where the initial flattening is depicted in red and the final solution is depicted in green.

Pseudocode corresponding to the complete flattening procedure is shown in Algorithm 1

\section{2. $2 D$ template definition}

\section{Overview}

To define the size and position of the PV and LAA circumferences so that they reflect their physiological relative location as much as possible, we analysed 67 


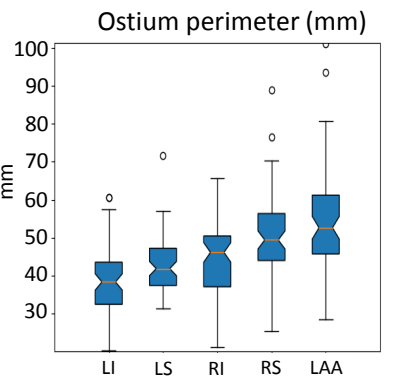

(a)

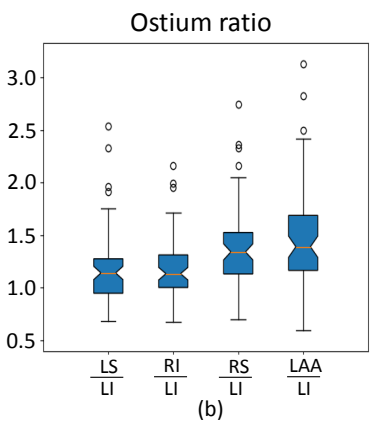

(b)

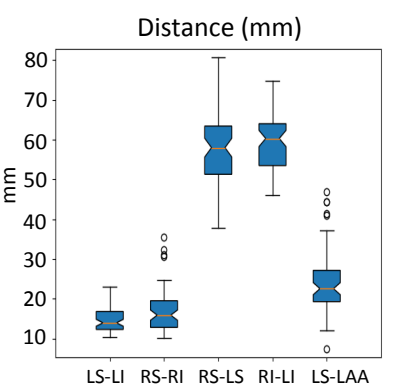

(c)

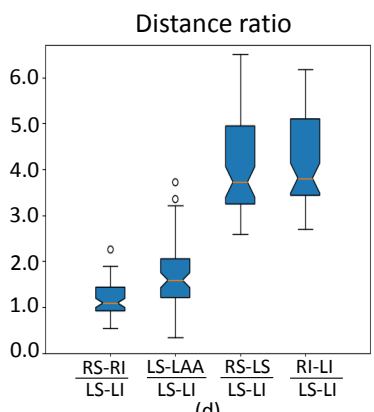

(d)

Figure 5: Summary of shape measurements. (a) PV and LAA ostium perimeters; (b) ratio between remaining ostium perimeters and LIPV perimeter that is the smallest one and considered as reference; (c) inter PV and LAA distances; (d) ratio of the inter PV and LAA distances and the LSPVLIPV separation (left carina) which is considered as reference. All distances are reported in $\mathrm{mm}$. LI = left inferior PV; LS = left superior PV; RI = right inferior PV; RS = right superior PV; LAA = left atrial appendage.

manually segmented LA (image resolution of $0.625 \mathrm{x}$ $0.625 \times 0.625 \mathrm{~mm}^{3}$ ) from the 2018 Atrial Segmentation Challeng $\mathrm{A}^{1}$ characterised by 4 separate PVs. The remaining atria from the challenge (33) presented different number of PVs or merged LAA and LSPV due to segmentation errors or imaging limitations. We first homogenised the meshes by clipping the PVs, LAA and MV using the procedure proposed by Tobon-Gomez et al. (2015). We measured the perimeter of PVs and LAA ostia; left and right carina lengths (i.e. distance between ipsilateral (same side) veins $\left(\mathrm{s}_{1}\right.$ and $\mathrm{s}_{3}$ in Figure 11); distance between the 2 inferior and the 2 superior veins, respectively $\left(\mathrm{s}_{2}\right.$ and $\mathrm{s}_{4}$ ); distance between LSPV and LAA ( $\mathrm{s}_{5}$ ); and distance between right and left veins and the MV $\left(\mathrm{s}_{6}-\mathrm{s}_{9}\right)$. We also used the 67 shapes to build a mean shape or template (shown in Figure 3) using Deformetrica (Routier et al., 2015).

Besides considering a realistic relative position of PVs and LAA, we additionally aimed to reduce distortion in the disk and achieve intuitive visualization. The small MV contour is mapped to a fairly bigger region, i.e. the contour of the disk, while at the same time constraining the remaining surface to the interior of the disk. This fact naturally induces stretching of the outer part of the disk and shrinkage of the interior (see Figure 3 a) causing high compression of information in the centre. Because of that, we created two additional templates modifying the measured PV and LAA relative positions to enlarge the central part of the disk and achieve better visualization.

An ideal 3D-2D mapping should transform the mesh elements (i.e. triangles) in an isotropic way and preserving their area. Therefore, to analyse the quality of the different LA unfolded maps, we defined distortion

\footnotetext{
${ }^{1}$ http://atriaseg2018.cardiacatlas.org/
}

indices based on area preservation and transformation isotropy. To quantify the area preservation we first normalised the size of the 3D and the corresponding 2D LA surfaces to have areas equal to 1 . Then, we computed for each triangle the ratio between its area in the 2D disk and in the 3D surface (let $\boldsymbol{\alpha}$ be the ratio). As the total areas were previously normalised, having $\alpha$ bigger than 1 implies triangle enlargement, smaller than 1 implies compression and equal to 1 reflects perfect size preservation. Constant ratio in the whole mesh is desired since it means that all triangles are equally transformed in terms of size. To inspect the isotropy of the transformation we used the Jacobian matrix of the 3D2D transformation (Lévy et al., 2002):

$$
\boldsymbol{J}=\left(\begin{array}{ll}
\partial u / \partial x & \partial u / \partial y \\
\partial v / \partial x & \partial v / \partial y
\end{array}\right)
$$

where $(x, y)$ are point coordinates in the local orthonormal coordinate system of each triangle and $(u, v)$ are the global coordinates of the flat domain. The two eigenvalues of $\boldsymbol{J}$ characterise the deformation of the triangle with the isotropy estimated as the ratio between the smallest and largest eigenvalue (let $\beta$ be the ratio, $\beta=$ 1 meaning isotropic transformation, and decreasing $\beta$ meaning anisotropic triangle stretching).

We additionally considered the Spatial Context Preservation (SCP), defined in Kreiser et al. (2018) as a qualitative index that helps the user to contextualize the observed structures: the better the spatial context is preserved, the easier a certain region can be visually related to its corresponding position in the original 3D data. To inspect the SCP of the different templates we created a synthetic texture pattern of same size circles uniformly distributed on the 3D surface mesh and inspected how the circles were displayed in the different templates. 


\section{Template (FRF map) creation}

Measurements corresponding to the PV and LAA ostium perimeters can be seen in Figure $5 \mathrm{a}$ and $\mathrm{b}$, and in the Appendix (Table A.1). According to our results the left PVs have significantly smaller perimeter than the right PVs $\left(p<10^{-6}\right)$. In particular, perimeters were related as follows: LIPV <* LSPV $<$ RIPV $<*$ RSPV $<$ LAA where $<^{*}$ denotes significantly smaller at the $5 \%$ significance level. Taking as a reference the smallest perimeter (LIPV) we established the following perimeter proportional values: $\mathrm{LSPV} / \mathrm{LIPV}=1.1$; RIPV/LIPV $=1.1 ; \mathrm{RSPV} / \mathrm{LIPV}=1.35 ;$ and LAA/LIPV $=1.35$, using population median values (Table A.1, right).

Measurements corresponding to the inter-vein distances $\left(s_{1-4}\right)$ and LSPV-LAA distance $\left(s_{8}\right)$ are summarized in Figure 55 and $d$, and in the Appendix (Table A.2). The left carina (i.e. region between the two left veins) was found significantly shorter than the right carina ( $p=0.007)$ with median ratio of 1.1 . The separation between LSPV and LAA was found significantly higher that the left carina length $\left(p<10^{-14}\right)$ with the median proportional ratio of almost 1.6. The separation between the 2 superior and the 2 inferior veins was found comparable $(p=0.37)$ and significantly higher than the carinas $\left(p<10^{-80}\right)$. The proportional ratio was estimated as 3.75 approximately (width of the posterior wall with respect to left carina length). Finally, we also found that the distance between left PVs and the MV contour was approximately half of the distance between right PVs and the MV contour.

Once all the ratios were estimated, the remaining step required to define the reference values (i.e. centre and radio of the LIPV circumference, and LIPV-LSPV (left carina) separation). Here the criterion used was to obtain a representation with low and uniform distortion distribution. We considered the SUM proposed by Williams et al. (2017) as the state of the art method and compared it with 3 different template (or FRF map) configurations:

1. Population-based: using the proportions measured in the study described above.

2. Adapted $_{1}$ : measured proportions are modified to achieve a more detailed representation of the central part of the disk (i.e. inter-veins region).

3. Adapted $_{2}$ : measured proportions are further modified to achieve SUM-like hole positions.

\section{Experiments and Results}

\subsection{Distortion analysis of $2 D$ templates}

Since the SUM methodology registers any LA to the $3 \mathrm{D}$ version of a template and then maps the information to the $2 \mathrm{D}$ version of the template, only that mesh (the template) can be fairly compared to our method. Results of flattening the SUM template can be seen in Figure 6 where the first row corresponds to the SUM mapping and the other 3 rows to the different FRF maps tested: Population-based, Adapted $_{1}$, and Adapted 2 . The different columns show, from left to right: the flattened mesh; the spatial distribution of the two distortion metrics (triangle's area ratio, $\alpha$, and anisotropy of the transformation, $\beta$ ); uniform circles mapping; and histograms of the distortion metrics considered. As it can be seen, SUM showed good triangle size preservation (triangles' area ratio close to 1) but with less compact histogram than the FRF maps where even if the difference between enlarged and compressed regions seemed emphasized (b), the histogram showed that the ratio between the areas was more constant than in the SUM case (most of the triangles were consistently reduced and only few triangles were enlarged). The SUM mapping had the worst anisotropic distortion profile (less percentage of values close to 1 (f)), especially close to the outer contour of the disk (c). FRF maps showed less anisotropic distortion specially for Population-based and Adapted 1 cases.

With regard to the comparison between the different FRF maps using the SUM template, Population-based and Adapted $_{1}$ showed comparable isotropic and area ratio profiles. However, the enlargement of the central

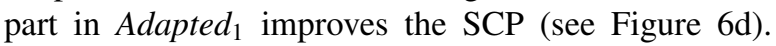
Adapted $_{2}$ was worse with regard to all the considered parameters showing higher anisotropic distortion and worse areas ratio resulting in an excessive and artificial vertical stretching of the central part. To decide between the 3 proposed FRF maps, we computed distortion metrics using a cohort of 15 LA. Figure 7 a shows boxplots of the anisotropic distortion metric corresponding to the 15 cases together and, as it can be seen, the three configurations showed comparable results. To determine the template with most compact triangle's size change, we compared the entropy of each histogram. Lower entropy indicates more stable triangle size change which is preferred in this context. As can be seen in Figure $7 \mathrm{p}$, the distortion is again comparable among the 3 configurations. We chose Adapted As $_{1}$ as the best compromise between isotropic transformation, triangle's area consistency and visual interpretability.

The regional relation between the final FRF map chosen $\left(\right.$ Adapted $\left._{1}\right)$ and the SUM was also studied, and it can 

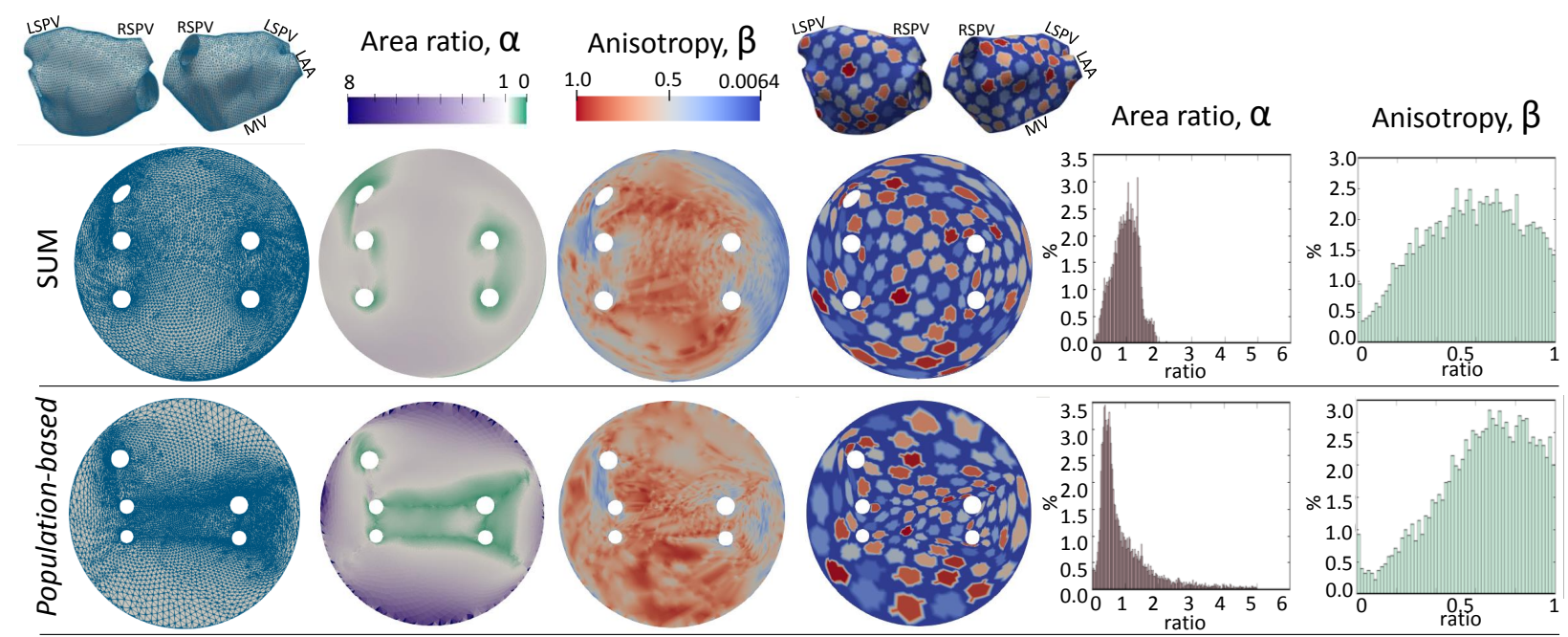

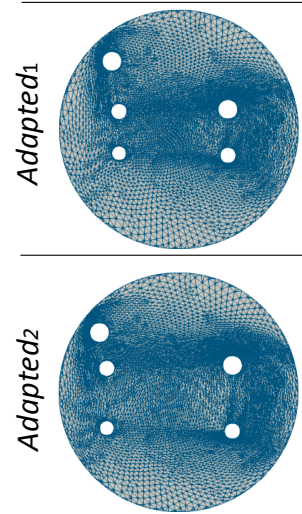

(a)

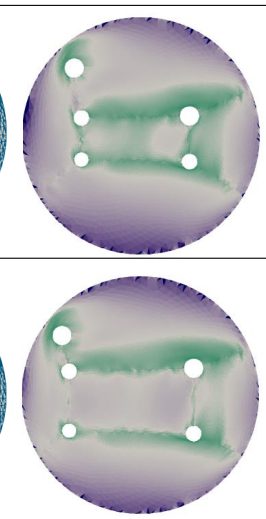

(b)

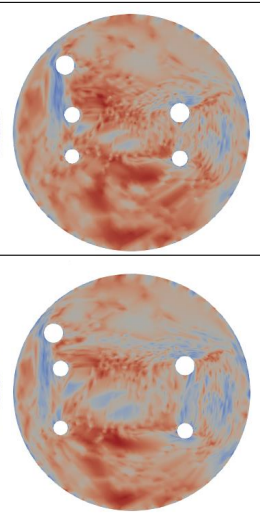

(c)

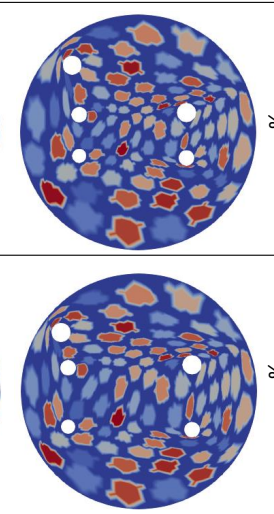

(d)
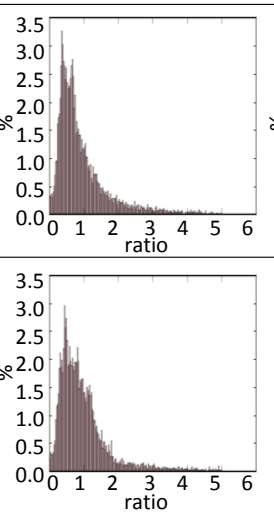

(e)

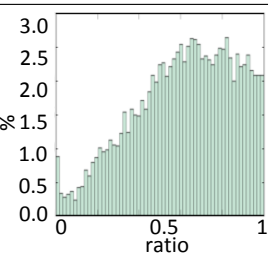

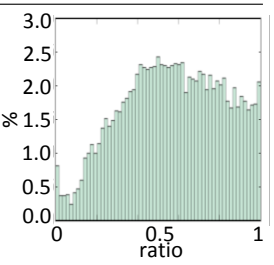

(f)

Figure 6: Distortion analysis performed using the 3D SUM template. The first row corresponds to the SUM template, and the other 3 to different FRF maps with different hole positions, population-based (second row), Adapted 1 (third row) and Adapted $_{2}$ (fourth row). (a) displays the mesh cells (triangles), (b) shows the area-related distortion, (c) the anisotropic distortion metric, (d) the result of mapping uniform circles, (e) histogram of $2 \mathrm{D} / 3 \mathrm{D}$ areas ratio $(\alpha)$, and (f) histogram of eigenvalues ratio $(\beta)$.

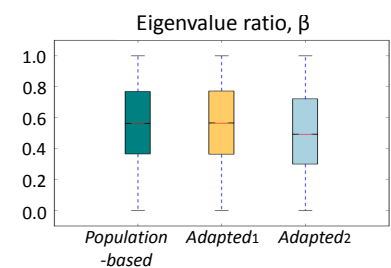

(a)

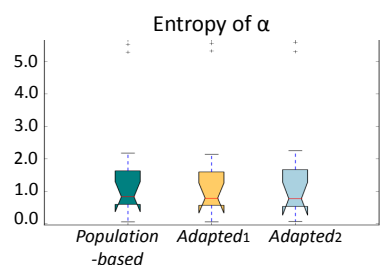

(b)
Figure 7: Distortion metrics in 15 LA. Distortion metrics do not significantly change depending on the templates.

be seen in Figure 8 a how the same regions are placed in different areas in the two 2D disks. We also used synthetic texture patterns to inspect the nature of the deformations induced by the FRF and compare it to the SUM mapping. Figure $8 \mathrm{~b}$ shows a striped pattern representing isocontours of the distance to a point placed in the centre of the region between the 4 veins. Figure 8 ; shows results of mapping a spotted pattern. As it can be seen, circles placed close to the MV appear highly distorted (stretched) in the SUM (e.g. spots number 1, 2, 6 or 8) while the effect of the regional flattening can be observed in the FRF map: note the orientation change of the spots in the region encircled in red, and the different size and shape of spots 8 and 9 (that should be similar since they are close to each other) because they belong to regions R4 and R1, respectively. Importantly, SUM is affected by interpolation errors due to information mapping (in this case, color of the spot) from the 3D LA to the SUM template (note the resolution loss as the circles appear blurred, e.g. in the region encircled in red, and the background color change). On the contrary, using the FRF method, there is no information loss or interpolation errors since all the points in the 3D mesh 

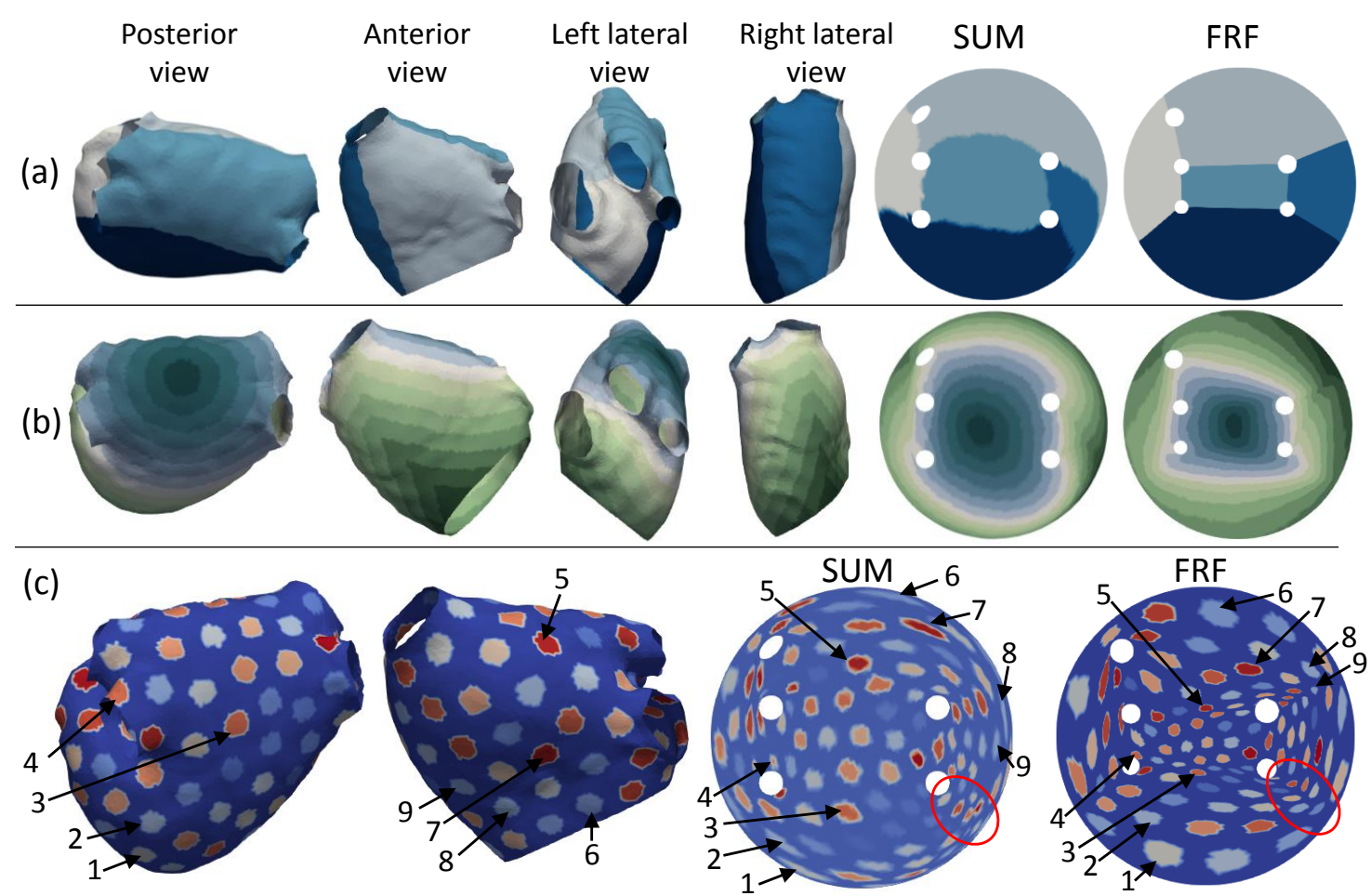

Figure 8: Flattening examples of 3D LA with different synthetic texture patterns on the surface. From top to bottom: 4 views of a sample LA divided into the 5 anatomical regions considered in our approach and regional correspondence to SUM and FRF maps (a); striped synthetic texture, defined on the 3D surface representing isocontours of the distance to a seed point placed in the posterior wall (b); and synthetic texture of 100 uniformly distributed spots (c). Some spots have been numbered in the different representations for clarification purposes.

are represented in the 2D map. Additional synthetic examples can be seen in the Appendix (Figure B.1).

\section{Clinical applications}

Several examples of FRF applied to the visualization of LGE-CMR image intensities are shown in the Appendix (Figure B.2). Besides the usefulness of the method for visualization purposes, in the following subsections, we present two clinical applications: quantification of incomplete ablation lines (gaps) after pulmonary vein isolation (PVI) and joint analysis of bipolar voltage information from electroanatomical maps (EAM) and LGE-MRI intensity data.

\subsection{Gap quantification.}

PVI is a common procedure for the treatment of AF that aims to electrically isolate the PVs from the LA body producing (with radiofrequency, laser or cryoballoon) a continuous lesion completely encircling the PVs. The resulting lesion is often incomplete being a combination of scar and gaps of healthy tissue that are potential causes for AF recurrence. We have previously developed a semi-automatic method to locate and quantify ablation gaps (Nuñez-Garcia et al., 2018) once a parcellation of the LA is available. Different parcellations can be defined in our FRF map and used to consistently divide different 3D LA in order to apply the gap quantification method. An example of the procedure can be seen in Figure 9.1. To identify gaps after PVI, the surroundings of the different PVs independently (Figure 9. 1 , top row) or in pairs (Figure 9 - 1 bottom row) must be inspected. The regions where the gaps are sought for were first defined in a high resolution FRF map used as template and were then transferred to the arbitrary FRF map using a closest point mapping and to the 3D LA using the mesh point identifier. After that, the automatic detection and quantification of the gaps was performed as described in Nuñez-Garcia et al. (2018).

\subsection{Inspection of the relation between EAM and $L G E$ - CMR data}

The proposed FRF maps can also be used to inspect multi-modal data from the same patient. The relation between voltage in EAM and signal intensity in LGECMR data has been investigated providing contradic- 


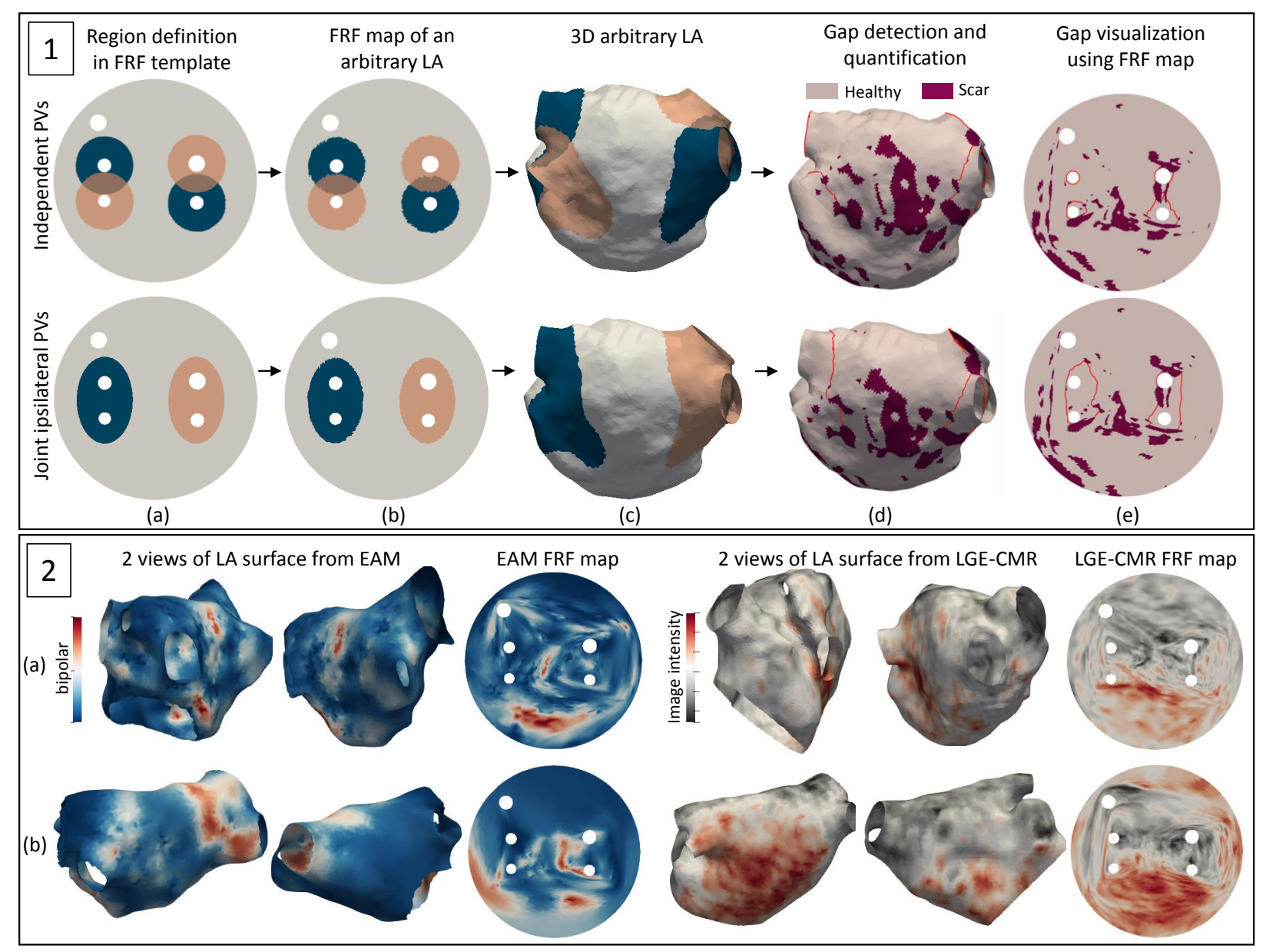

Figure 9: Examples of clinical applications of FRF. 1. Gap quantification using the FRF method: from left to right: sub-regions are defined in a high resolution FRF map template as the surrounding of each PV (top row) or the surroundings of the two ipsilateral (same-side) PVs (bottom row); the defined subdivision is transferred to the FRF map obtained from an arbitrary LA (b) and then to the corresponding 3D arbitrary LA (c); automatic detection and quantification of gaps (in red) is performed on the 3D LA (d) and optionally displayed on the disk (e). 2. Two examples of electroanatomical maps (EAM) and corresponding (same patient) LA from LGE-CMR with projected image intensity. Low voltage (dark blue) is typically associated to high LGE-CMR intensity (red). LGE-CMR = Late Gadolinium Enhanced Cardiac Magnetic Resonance.

tory results (Spragg et al., 2012; Bisbal et al., 2014 . Harrison et al. 2015). Figure 9-2 shows two examples of EAM invasively acquired during an electrophysiology study (using CARTO (Biosense Webster Inc, Diamond Bar, CA) mapping system) and corresponding (i.e. same patient) LA segmented from LGE-CMR with image intensity projected onto the surface. More examples are shown in the Appendix (Figure B.3). It can be seen how FRF puts into correspondence the two different (due to the different acquisition methods) LA anatomies facilitating their qualitative and quantitative comparison.

\section{Discussion}

We have proposed a novel, fast and information lossless LA flattening framework that could be also applied to other similar structures (e.g. the right atria) only requiring the adaptation of the template (boudary and regional constraints) to the specific structure's anatomy.

We thoroughly compared our method to the SUM mapping, the state of the art method for atrial standardised representation. Contrary to the SUM method, FRF does not depend on registration being therefore faster and not affected by potential registration errors. Additionally, FRF does not lose any information from the initial 3D surface, i.e. all points are depicted in the 2D map, while SUM needs to interpolate data when map- 
ping the information from the arbitrary 3D surface to the template. This is especially noticeable in the flattening of the spotted patterns (color represents spot identifier) where the texture appears blurred after the flattening process (Figure 6). This effect is even more noticeable in the example in Appendix (Figure B.1.) where it can be seen how many spots have merged due to resolution loss. These errors could be reduced by remeshing the SUM template (increasing the number of points) but this will increase the registration computational cost. The execution time of the registration using the original SUM template (mesh with 7402 points) is around 20 minutes (Nuñez-Garcia et al. 2018) while FRF lasts 2-3 s, after manual seed points placement (Intel i5 3.3 GHz CPU and 16 GB RAM).

The distortion induced by the two methods is different as showed in Figure 6 SUM method performs a global mapping where the central part is enlarged and the areas close to the MV are pushed and stretched along the contour of the disk. On the other hand, FRF restricts each area to its specific 2D domain (enhancing regional interpretability) even if this fact causes local stretching/shrinkage and distortion close to the limits of the 5 defined regions.

SUM provides more detailed regional division (24 regions (Williams et al., 2017) vs. our 5 regions) but this regional division may be inaccurate due to the small size of some regions and the influence of registration errors. FRF brings more control on the 3D regional division by manual placement of seed points and, in the flattening, constraining each region to its specific 2D area. Our algorithm is however highly influenced by this manual seed placement step, especially regarding the seeds placed on the MV contour. The seed points and inter-seed paths define the constraints used in the flattening which is the key part of the method. Automatically computing the constraints, i.e. automatically dividing the LA is challenging due to, for example, bulges in the LA and obliqueness of the MV plane. Nonetheless, since the process of seed placement and unfolding is almost real time it can be repeated several times until a satisfactory result is obtained. Additionally, more regions could be defined in our FRF template to further subdivide any arbitrary LA as in Figure 9 - 1 a-c.

Side-by-side or overlaying inspection of different FRF maps is convenient in many clinical situations: to analyse the temporal evolution of some parameter (e.g. pre- and post-ablation fibrosis extent) from the same patient; to compare and correlate different features from the same patient (e.g. gadolinium enhancement from LGE-CMR data and endocardial voltage from electroanatomical maps); to compare same feature in differ- ent patients (e.g. regional or global fibrosis extent (Benito et al. 2018), etc. We have shown pairwise comparisons of LGE-CMR intensities and bipolar endocardial voltage but a better depiction of the electroanatomical maps is required (e.g. using high definition mapping systems like Rhythmia (HDx Mapping System, Boston Scientific)) to achieve realistic representations of the LA and be able to fairly compare it with other kinds of data such as LGE-MRI.

Proposing a standard 2D template is far from trivial. We observed how depending on the specific 3D atrial shape, different configurations (mainly radius and position of the holes) were preferable. We developed a template that can be used for most of the LA shapes (with 4 veins), which takes into account the real averaged inter-vein (and LAA) separation and facilitates visualization and interpretability of the 2D map. However, the estimated metrics (proportions) were obtained using a dataset of AF patients, i.e. bigger, more spherical LA and with bigger PV ostia than controls. WoźniakSkowerska et al. (2011) showed how diameters of PV ostia were larger in AF patients than in control subjects in accordance with increased LA volume of the AF patients. Additionally, Lickfett et al. (2005) found that the PV orifice size depended on the cardiac cycle with the largest diameter in late atrial systole with a mean reduction of $32.5 \%$ in atrial systole. Nonetheless, we believe that the changes in the size of the atrium or ostia will not change significantly our distortion analysis.

The method presents some limitations. First, it can only be applied to LA with 4 PVs. Other templates can be derived similarly to the one proposed here but direct comparison with the 4-veins template will not be accurate in regions close to the PV holes. The method can be applied to atria with a common left trunk if there exist a distant bifurcation but it should be taken into account that the region represented between the veins would not be the carina but the common left trunk itself. LA with extra veins (e.g. extra middle right PV) can also be flattened with our method if the vein can be clipped or ignored. This solution may be acceptable depending on the application. For example, it could be done to quantify global extent of fibrosis but it should be avoided if the representation of the surroundings of the PVs is important, for example in gap detection after pulmonary vein isolation. Our method may provide unsatisfactory results in the case of low resolution meshes (few points) and with PVs very close to each other. In that case, the number of points between the veins may not be sufficient to depict the template and the triangles may appear stretched.

The code (Python) will be publicly available once the 
paper is published.

\section{Conclusion}

We have presented a method to unfold any 3D LA surface and depict it in an intuitive, standardised, twodimensional map. Compared to the SUM it does not rely on a registration step, much faster and guarantees no information loss, as it could happen due to a bad registration or information interpolation when the source and target meshes have very different shapes an resolutions. Moreover, it emphasizes regional interpretability by confining anatomical regions to predefined regions in the 2D map avoiding undesired displacements attributable to global flattening methods. We have shown the suitability of our method to easily inspect the whole LA anatomy in 1 view, and to consistently divide the different LA anatomies and put them into correspondence making its regional comparison possible.

\section{Acknowledgments}

This study was partially funded by the Spanish Ministry of Economy and Competitiveness (DPI2015-71640-R), by the "Fundació La Marató de TV3" ( ${ }^{\circ}$ 20154031) and by European Union Horizon 2020 Programme for Research and Innovation, under grant agreement No. 642676 (CardioFunXion). 


\section{Appendix A. Shape metrics.}

Table A.1: Mean, standard deviation (SD) and median of the PVs and LAA ostium perimeters (left) and perimeter ratio with respect to LIPV which is the smallest one and considered as reference (right). All distances are reported in mm. LIPV = left inferior PV; LSPV $=$ left superior PV; RIPV = right inferior PV; RSPV = right superior PV; LAA = left atrial appendage.

\begin{tabular}{cccccc|cccc}
\multicolumn{7}{c|}{ Perimeter $(\mathbf{m m})$} & \multicolumn{4}{c}{ Perimeters ratio } \\
\hline & LIPV & LSPV & RIPV & RSPV & LAA & $\frac{L S P V}{L I P V}$ & $\frac{R I P V}{L I P V}$ & $\frac{R S P V}{L I P V}$ & $\frac{L A A}{L I P V}$ \\
\hline Mean & 38.73 & 43.06 & 44.49 & 50.47 & 54.30 & 1.17 & 1.18 & 1.36 & 1.48 \\
SD & 9.17 & 7.37 & 9.91 & 11.21 & 14.06 & 0.35 & 0.29 & 0.39 & 0.50 \\
Median & 38.32 & 41.84 & 46.11 & 49.39 & 52.61 & 1.14 & 1.13 & 1.34 & 1.39
\end{tabular}

Table A.2: Mean, standard deviation and median of the inter-veins and LAA distance (left) and distance ratio with respect to the LSPV-LIPV (left carina) distance which is considered as reference (right). All distances are reported in mm. LIPV = left inferior PV; LSPV = left superior PV; RIPV = right inferior PV; RSPV = right superior PV; LAA = left atrial appendage.

\begin{tabular}{|c|c|c|c|c|c|c|c|c|c|}
\hline & \multicolumn{5}{|c|}{ Distance $(\mathbf{m m})$} & \multicolumn{4}{|c|}{ Distance ratio } \\
\hline & LSPV-LIPV & RSPV-RIPV & RSPV-LSPV & RIPV-LIPV & LSPV-LAA & $\frac{R S P V-R I P V}{L S P V-L I P V}$ & $\frac{L S P V-L A A}{L S P V-L I P V}$ & $\frac{R S P V-L S P V}{L S P V-L I P V}$ & $\frac{R I P V-L I P V}{L S P V-L I P V}$ \\
\hline Mean & 14.82 & 17.03 & 57.66 & 58.91 & 23.94 & 1.18 & 1.72 & 4.06 & 4.15 \\
\hline SD & 3.29 & 5.62 & 8.61 & 7.21 & 7.95 & 0.36 & 0.75 & 1.01 & 0.99 \\
\hline Median & 13.96 & 15.88 & 57.98 & 60.29 & 22.54 & 1.09 & 1.59 & 3.72 & 3.81 \\
\hline
\end{tabular}

Appendix B. Additional flattening examples.

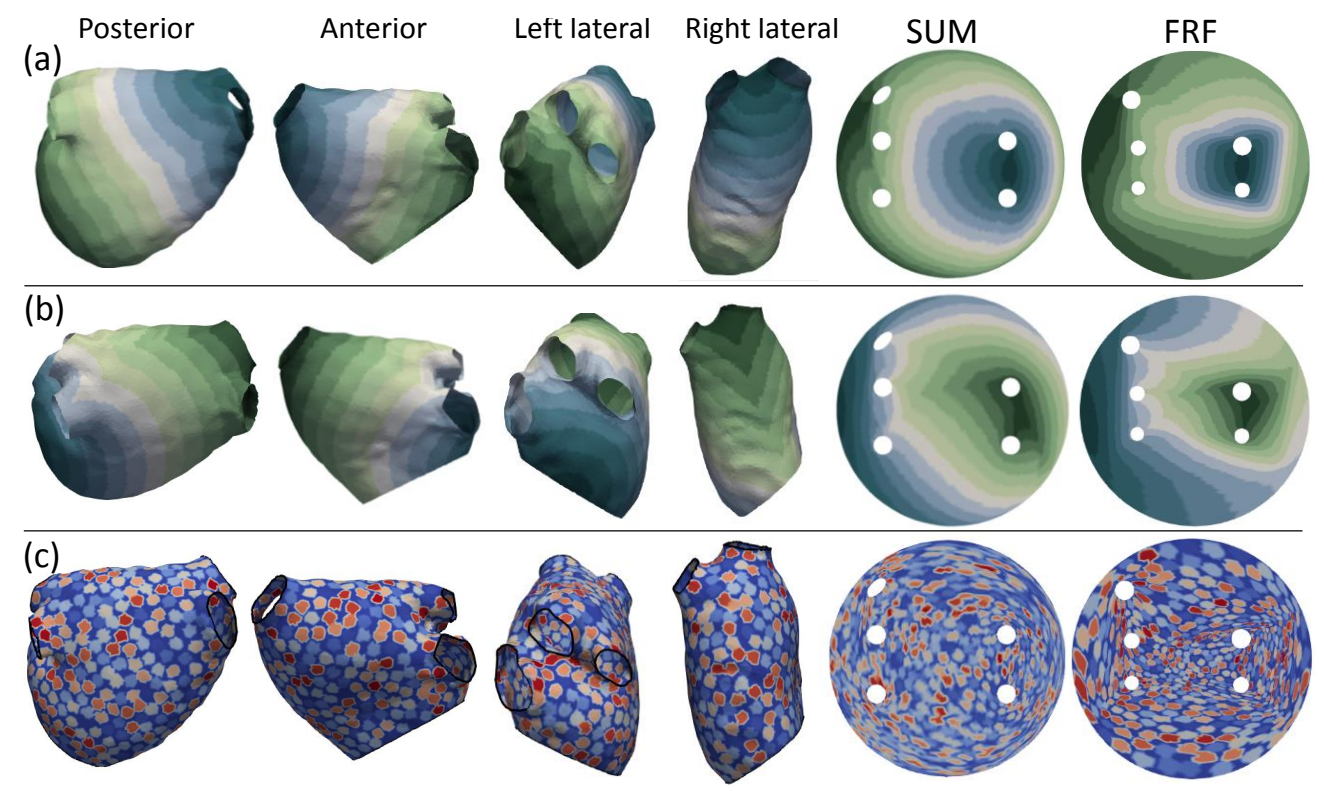

Figure B.1: Additional flattening examples of 3D LA with different synthetic textures on the surface and corresponding SUM and FRF maps. First and second rows show striped synthetic patterns, defined on the $3 \mathrm{D}$ surface representing isocontours of the distance to a seed point placed in the right carina (a), and the MV contour (b). (c) shows a texture of 500 uniformly distributed spots. 
(a)

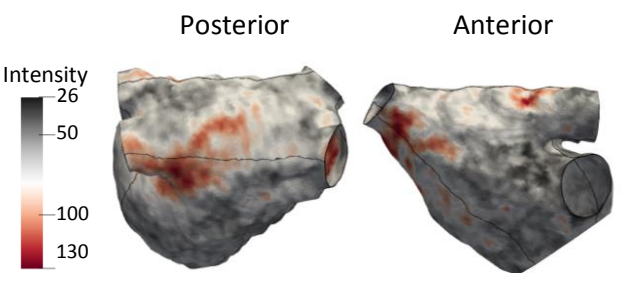

(b)
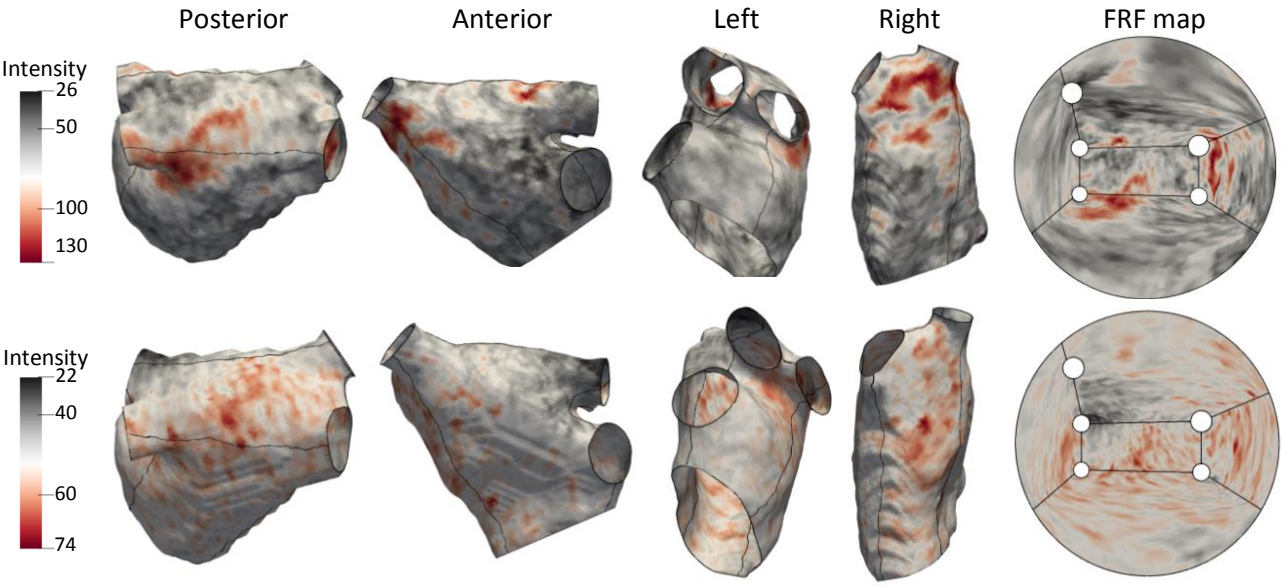

(c)
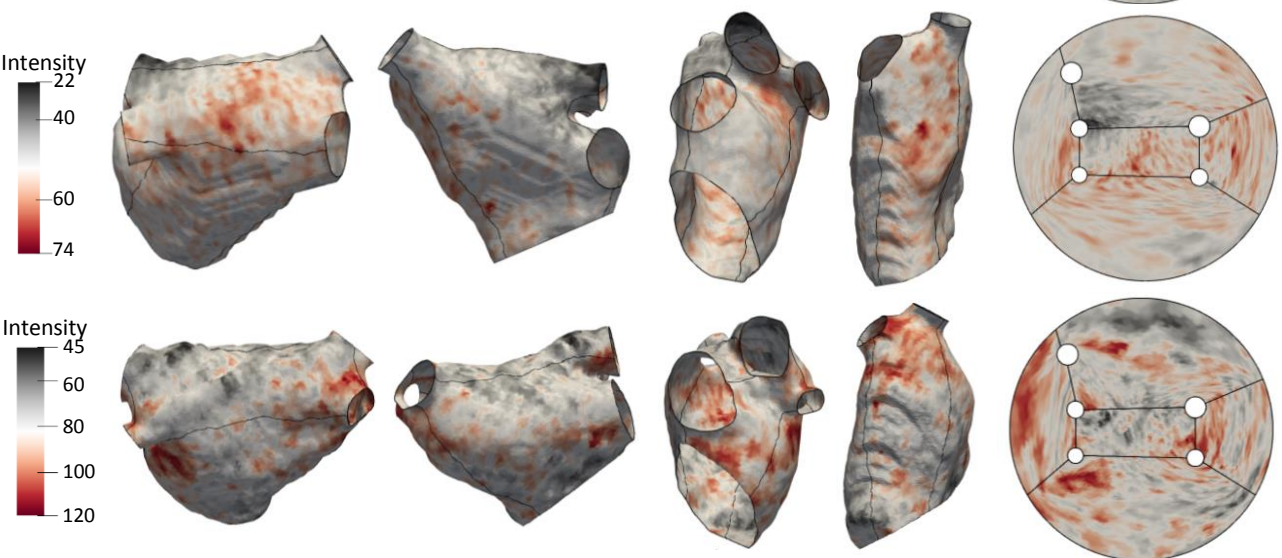

(d)
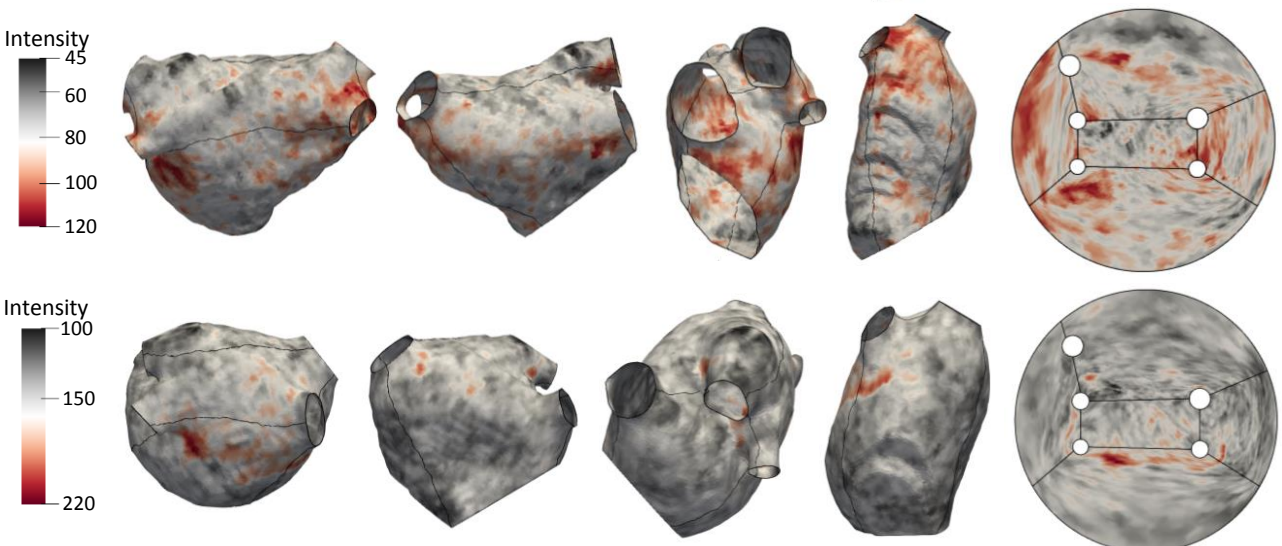

Figure B.2: Examples of LA flattening showing 4 views of manually segmented LA surfaces with projected late gadolinium enhanced magnetic resonance imaging (LGE-CMR) intensities and corresponding FRF maps.
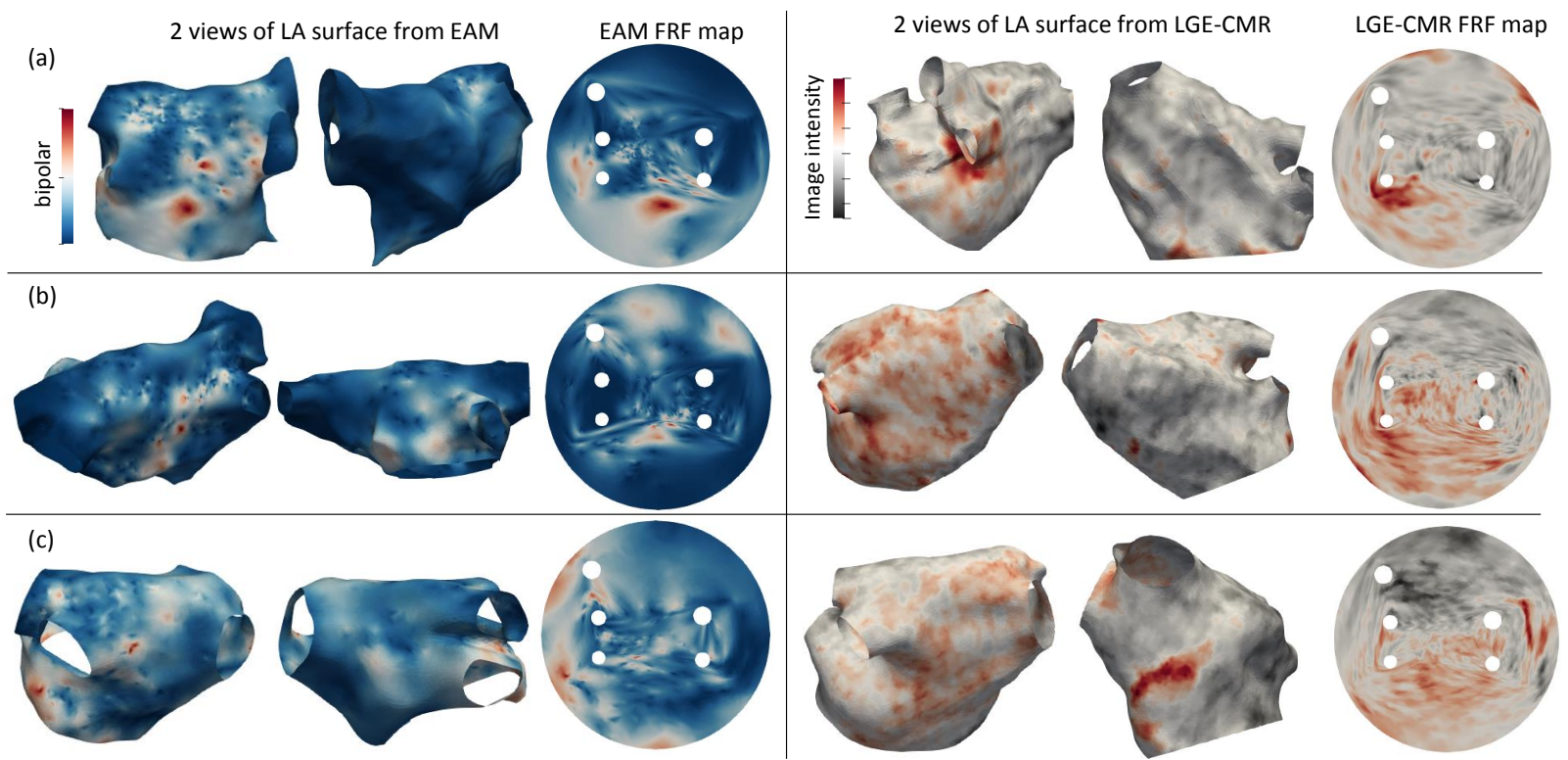

Figure B.3: Three examples of EAM and corresponding (same patient) LA from LGE-CMR with projected image intensity. Low voltage (dark blue) is typically associated to high LGE-CMR intensity (red). 


\section{References}

Benito, E. M., Cabanelas, N., Nuñez-Garcia, M., Alarcón, F., Ventura, F. I., Rosa, M., Soto-Iglesias, D., Guasch, E., Prat-Gonzalez, S., Perea, R. J., et al., 2018. Preferential regional distribution of atrial fibrosis in posterior wall around left inferior pulmonary vein as identified by late gadolinium enhancement cardiac magnetic resonance in patients with atrial fibrillation. EP Europace.

Bisbal, F., Guiu, E., Cabanas-Grandío, P., Berruezo, A., PratGonzalez, S., Vidal, B., Garrido, C., Andreu, D., FernandezArmenta, J., Tolosana, J. M., et al., 2014. CMR-guided approach to localize and ablate gaps in repeat $\mathrm{AF}$ ablation procedure. JACC: Cardiovascular Imaging 7 (7), 653-663.

Cerqueira, M. D., Weissman, N. J., Dilsizian, V., Jacobs, A. K., Kaul, S., Laskey, W. K., Pennell, D. J., Rumberger, J. A., Ryan, T., Verani, M. S., et al., 2002. Standardized myocardial segmentation and nomenclature for tomographic imaging of the heart: a statement for healthcare professionals from the cardiac imaging committee of the council on clinical cardiology of the american heart association. Circulation 105 (4), 539-542.

García-Isla, G., Olivares, A. L., Silva, E., Nuñez-Garcia, M., Butakoff, C., Sanchez-Quintana, D., G. Morales, H., Freixa, X., Noailly, J., De Potter, T., et al., 2018. Sensitivity analysis of geometrical parameters to study haemodynamics and thrombus formation in the left atrial appendage. International journal for numerical methods in biomedical engineering 34, e3100.

Harrison, J. L., Sohns, C., Linton, N. W., Karim, R., Williams, S. E., Rhode, K. S., Gill, J., Cooklin, M., Rinaldi, C. A., Wright, M., et al., 2015. Repeat left atrial catheter ablation: cardiac magnetic resonance prediction of endocardial voltage and gaps in ablation lesion sets. Circulation: Arrhythmia and Electrophysiology 8 (2), 270-278.

Karim, R., Ma, Y., Jang, M., Housden, R. J., Williams, S. E., Chen, Z., Ataollahi, A., Althoefer, K., Rinaldi, C. A., Razavi, R., et al., 2014. Surface flattening of the human left atrium and proof-ofconcept clinical applications. Computerized Medical Imaging and Graphics 38 (4), 251-266.

Kreiser, J., Meuschke, M., Mistelbauer, G., Preim, B., Ropinski, T., 2018. A Survey of Flattening-Based Medical Visualization Techniques. In: Computer Graphics Forum. Vol. 37. No. 3. pp. 597624.

Lévy, B., Petitjean, S., Ray, N., Maillot, J., 2002. Least squares conformal maps for automatic texture atlas generation. In: ACM transactions on graphics (TOG). Vol. 21, No. 3. pp. 362-371.

Lickfett, L., Dickfeld, T., Kato, R., Tandri, H., Vasamreddy, C. R., Berger, R., Bluemke, D., LÜderitz, B., Halperin, H., Calkins, H., 2005. Changes of pulmonary vein orifice size and location throughout the cardiac cycle: dynamic analysis using magnetic resonance cine imaging. Journal of cardiovascular electrophysiology 16 (6), 582-588.

Liepa, P., 2003. Filling holes in meshes. In: Proceedings of the 2003 Eurographics/ACM SIGGRAPH symposium on Geometry processing. Eurographics Association, pp. 200-205.

Ma, Y., Karim, R., Housden, R. J., Gijsbers, G., Bullens, R., Rinaldi, C. A., Razavi, R., Schaeffter, T., Rhode, K. S., 2012. Cardiac unfold: a novel technique for image-guided cardiac catheterization procedures. In: International Conference on Information Processing in Computer-Assisted Interventions. pp. 104-114.

Mitchell, J. S., Mount, D. M., Papadimitriou, C. H., 1987. The discrete geodesic problem. SIAM Journal on Computing 16 (4), 647-668.

Nuñez-Garcia, M., Camara, O., O’Neill, M. D., Razavi, R., Chubb, H., Butakoff, C., 2018. Mind the gap: quantification of incomplete ablation patterns after pulmonary vein isolation using minimum path search. Medical Image Analysis. In press.

Paun, B., Bijnens, B., Iles, T., Iaizzo, P. A., Butakoff, C., 2017. Pa- tient independent representation of the detailed cardiac ventricular anatomy. Medical image analysis 35, 270-287.

Pinkall, U., Polthier, K., 1993. Computing discrete minimal surfaces and their conjugates. Experimental mathematics 2 (1), 15-36.

Prasanna, L., Praveena, R., AS, D., Kumar, M., 2014. Variations in the pulmonary venous ostium in the left atrium and its clinical importance. Journal of clinical and diagnostic research 8 (2), 10.

Routier, A., Prastawa, M., Charlier, B., Doucet, C., Glaunes, J. A., Durrleman, S., 2015. Deformetrica: a software for statistical analysis of anatomical shapes. In: 21st Annual Meeting of the Organization for Human Brain Mapping, OHBM 2015.

Soto-Iglesias, D., Butakoff, C., Andreu, D., Fernández-Armenta, J., Berruezo, A., Camara, O., 2016. Integration of electro-anatomical and imaging data of the left ventricle: an evaluation framework. Medical image analysis 32, 131-144.

Spragg, D. D., Khurram, I., Zimmerman, S. L., Yarmohammadi, H., Barcelon, B., Needleman, M., Edwards, D., Marine, J. E., Calkins, H., Nazarian, S., 2012. Initial experience with magnetic resonance imaging of atrial scar and co-registration with electroanatomic voltage mapping during atrial fibrillation: success and limitations. Heart Rhythm 9 (12), 2003-2009.

Tobon-Gomez, C., Geers, A. J., Peters, J., Weese, J., Pinto, K., Karim, R., Ammar, M., Daoudi, A., Margeta, J., Sandoval, Z., et al., 2015. Benchmark for algorithms segmenting the left atrium from 3D CT and MRI datasets. IEEE transactions on medical imaging 34 (7), 1460-1473.

Williams, S. E., Tobon-Gomez, C., Zuluaga, M. A., Chubb, H., Butakoff, C., Karim, R., Ahmed, E., Camara, O., Rhode, K. S., 2017. Standardized unfold mapping: a technique to permit left atrial regional data display and analysis. Journal of Interventional Cardiac Electrophysiology 50 (1), 125-131.

Woźniak-Skowerska, I., Skowerski, M., Wnuk-Wojnar, A., Hoffmann, A., Nowak, S., Gola, A., Sosnowski, M., Trusz-Gluza, M., 2011. Comparison of pulmonary veins anatomy in patients with and without atrial fibrillation: analysis by multislice tomography. International journal of cardiology 146 (2), 181-185. 